\title{
Existence of symmetric solutions for a class of BVP with integral boundary conditions
}

\section{Zhiying Tong and Wei Ding*}

\section{"Correspondence:} dingwei@shnu.edu.cn Department of Mathematics, Shanghai Normal University, Shanghai, 200234, P.R. China

\begin{abstract}
In this paper, we study the symmetric solutions of second-order BVP with integral boundary conditions. By using a generalized Leggett-Williams fixed point theorem and some other techniques, we obtain sufficient conditions for the existence of symmetric positive solutions for the system. Meanwhile, an example is devoted to demonstrate our results in the end.
\end{abstract}

Keywords: integral boundary conditions; Green's function; boundary value problem; fixed point theorem

\section{Introduction}

With the development of science and technology, boundary value problems have acquired more attention in these years. Many methods are used to solve this kind of problems, such as fixed point theorems, coincidence degree theory, iterative method with upper and lower solutions, etc. Readers can see [1-13] for details.

However, the past researches focus on the existence of positive solution, periodic solution, uniqueness, etc.

For example, Boucherif [14] considered the following BVP:

$$
\left\{\begin{array}{l}
u^{\prime \prime}(t)+f(u(t))=0, \quad t \in(0,1), \\
u(0)-a u^{\prime}(0)=\int_{0}^{1} g_{0}(s) u(s) d s \\
u(1)-b u^{\prime}(1)=\int_{0}^{1} g_{1}(s) u(s) d s .
\end{array}\right.
$$

By applying the Krasnoselskii fixed point theorem, they obtained the existence of positive solutions of the system.

After Hayasida [15] drew some interesting results about symmetric positive solutions for a kind of BVP, several papers concerned about the subject with the same method; see [16-19]. Inspired by them, we consider a similar problem for the following system:

$$
\left\{\begin{array}{l}
u^{\prime \prime}(t)+f(u(t))=0, \quad t \in I \\
u(0)-a u^{\prime}(0)=\int_{0}^{1} \varrho_{0}(s) u(s) d s \\
u(1)-b u^{\prime}(1)=\int_{0}^{1} \varrho_{1}(s) u(s) d s
\end{array}\right.
$$

(c) 2016 Tong and Ding. This article is distributed under the terms of the Creative Commons Attribution 4.0 International License (http://creativecommons.org/licenses/by/4.0/), which permits unrestricted use, distribution, and reproduction in any medium, provided you give appropriate credit to the original author(s) and the source, provide a link to the Creative Commons license, and indicate if changes were made. 
where $f: \mathbb{R} \rightarrow[0,+\infty)$ is continuous, $I=[0,1], \varrho_{0}>0$ and $\varrho_{1}>0$ are continuous, and $a$ and $b$ are real parameters.

Abdulkadir [20] studied the case $a=b=0, \varrho_{0}, \varrho_{1} \equiv 0$ and drew some conclusions by using a generalization of the Leggett-Williams fixed point theorem. Obviously, the general case is much complicated. In this paper, we generalize the corresponding results.

The structure of this paper is as follows. In Section 2 and Section 3, we introduce the Leggett-Williams fixed point theorem, some definitions, and some lemmas. In particular, we deduce system (4) and some properties of the Green function, which will be used to prove the main results. Section 4 is devoted to developing the main results, which will be stated in detail. Finally, an example is included to display the main results.

\section{Preliminaries}

In this section, we give some definitions and the fixed point theorem that will be used in this paper.

Definition 1 Let $E$ be a real Banach space. A nonempty, closed, and convex set $P \subset E$ is a cone if the following two conditions are satisfied:

(i) if $x \in P$ and $\mu \geq 0$, then $\mu x \in P$;

(ii) if $x \in P$ and $-x \in P$, then $x=0$.

Every cone $P \subset E$ induces the ordering in $E$ given by $x_{1} \leq x_{2}$ if and only if $x_{2}-x_{1} \in P$.

Definition 2 A map $\alpha$ is called a nonnegative continuous convex functional on a cone $P$ in a real Banach space $E$ if $\alpha: P \rightarrow[0,+\infty)$ is continuous and

$$
\alpha\left(\lambda x_{1}+(1-\lambda) x_{2}\right) \leq \lambda \alpha\left(x_{1}\right)+(1-\lambda) \alpha\left(x_{2}\right)
$$

for all $x_{1}, x_{2} \in P$ and $0 \leq \lambda \leq 1$. Likewise, we know the map $\beta$ is a nonnegative continuous concave functional on a cone $P$ in a real Banach space $E$ if $\beta: P \rightarrow[0,+\infty)$ is continuous and

$$
\beta\left(\lambda x_{1}+(1-\lambda) x_{2}\right) \geq \lambda \beta\left(x_{1}\right)+(1-\lambda) \beta\left(x_{2}\right)
$$

for all $x_{1}, x_{2} \in P$ and $0 \leq \lambda \leq 1$.

We denote $E=C(I), I=[0,1]$, with the maximum norm, and for all $0<\tilde{t} \leq \frac{1}{2}$, we define the cone $P \subset E$ by

$$
\begin{aligned}
P= & \{u \in E: u(t) \text { is concave, symmetric, and nonnegative-valued on } I, \\
& \text { and } \left.\min _{t \in[\tilde{t}, 1-\widetilde{t}]} u(t) \geq 2 \widetilde{t}\|u\|\right\} .
\end{aligned}
$$

Theorem 1 (Leggett-Williams fixed-point theorem [21]) Let $P \subset E$ be a cone in a real Banach space E. Let $l>0$ and $N>0$, let $\beta$ and $\chi$ be nonnegative continuous concave functionals on $P$, and let $\zeta, \alpha$, and $\rho$ be nonnegative continuous convex functionals on $P$ with

$$
\beta(u) \leq \alpha(u), \quad\|u\| \leq N \zeta(u),
$$


for all $u \in \overline{\mathbb{P}(\zeta, l)}$. Suppose that $Y: \overline{\mathbb{P}(\zeta, l)} \rightarrow \overline{\mathbb{P}(\zeta, l)}$ is a completely continuous operator and that there exist numbers $h>0, d>0, p, q>0$ with $d<p$ such that:

$$
\begin{aligned}
& u \in \mathbb{P}(\zeta, \rho, \beta, p, q, l): \quad \beta(u)>p \neq \emptyset \quad \text { and } \quad \beta(F u)>p \quad \text { for } u \in \mathbb{P}(\zeta, \rho, \beta, p, q, l) ; \\
& u \in \mathbb{Q}(\zeta, \alpha, \chi, h, d, l): \quad \alpha(u)<d \neq \emptyset \quad \text { and } \quad \alpha(F u)<d \quad \text { for } u \in \mathbb{Q}(\zeta, \alpha, \chi, h, d, l) ; \\
& \alpha(F u)>p \quad \text { for } u \in \mathbb{P}(\zeta, \beta, p, l) \text { with } \rho(F u)>q ; \\
& \beta(F u)<d \quad \text { for } u \in \mathbb{Q}(\zeta, \alpha, d, l) \text { with } \chi(F u)<h .
\end{aligned}
$$

Then there exist at least three fixed points $u_{1}, u_{2}, u_{3} \in \overline{\mathbb{P}(\zeta, l)}$ such that

$$
\alpha\left(u_{1}\right)<d, \quad p<\beta\left(u_{2}\right), \text { and } d<\alpha\left(u_{3}\right) \quad \text { with } \beta\left(u_{3}\right)<p .
$$

Thereinto, some sets are as follows:

$$
\begin{aligned}
& \mathbb{P}(\zeta, l)=\{u \in P: \zeta(u)<l\}, \\
& \mathbb{P}(\zeta, \beta, p, l)=\{u \in P: p \leq \beta(u), \zeta(u)<l\}, \\
& \mathbb{P}(\zeta, \rho, \beta, p, q, l)=\{u \in P: p \leq \beta(u), \rho(u) \leq q, \zeta(u)<l\}, \\
& \mathbb{Q}(\zeta, \alpha, d, l)=\{u \in P: \alpha(u) \leq d, \zeta(u)<l\}, \\
& \mathbb{Q}(\zeta, \alpha, \chi, h, d, l)=\{u \in P: h \leq \chi(u), \alpha(u) \leq d, \zeta(u)<l\} .
\end{aligned}
$$

Many other functionals on the cone $P$ are defined by

$$
\begin{aligned}
& \beta(u)=\min _{t \in\left[t_{0}, t_{1}\right] \cup\left[1-t_{1}, 1-t_{0}\right]} u(t)=u\left(t_{0}\right), \\
& \chi(u)=\min _{t \in\left[\frac{1}{\omega}, \frac{\omega-1}{\omega}\right]} u(t)=u\left(\frac{1}{\omega}\right), \\
& \alpha(u)=\max _{t \in\left[\frac{1}{\omega}, \frac{\omega-1}{\omega}\right]} u(t)=u\left(\frac{1}{2}\right), \\
& \rho(u)=\max _{t \in\left[t_{0}, t_{1}\right] \cup\left[1-t_{1}, 1-t_{0}\right]} u(t)=u\left(t_{1}\right), \\
& \zeta(u)=\max _{t \in[0, \widetilde{t}] \cup[1-\widetilde{t}, 1]} u(t)=u(\widetilde{t}),
\end{aligned}
$$

where $t_{0}, t_{1}$, and $\omega$ are nonnegative numbers such that

$$
0<t_{0}<t_{1} \leq \frac{1}{2} \text { and } \quad \frac{1}{\omega} \leq t_{1}
$$

It is clear that, for all $u \in P$,

$$
\begin{aligned}
& \beta(u)=u\left(t_{0}\right) \leq u\left(\frac{1}{2}\right)=\alpha(u), \\
& \|u\|=u\left(\frac{1}{2}\right) \leq \frac{1}{2 \widetilde{t}} u(\widetilde{t})=\frac{1}{2 \widetilde{t}} \zeta(u) .
\end{aligned}
$$

Throughout the paper, we suppose that the following two conditions hold. 
$\left(H_{0}\right) \quad a>1,-1<b<0$;

$\left(H_{1}\right) \varrho_{0}>0$ and $\varrho_{1}>0$ are continuous on $I$, and the supplementary function $\varphi(t, s)$, defined by

$$
\varphi(t, s)=\frac{a+t}{1+a-b} \varrho_{1}(s)-\frac{b-1+t}{1+a-b} \varrho_{0}(s), \quad t, s \in I,
$$

satisfies

$$
0 \leq m:=\min _{t, s \in I} \varphi(t, s) \leq M:=\max _{t, s \in I} \varphi(t, s)<1
$$

\section{Some lemmas}

In order to get the main results, we consider the following linear system:

$$
\left\{\begin{array}{l}
-u^{\prime \prime}(t)=h(t), \quad t \in[0,1] \\
u(0)-a u^{\prime}(0)=\int_{0}^{1} \varrho_{0}(s) \sigma_{0}(s) d s \\
u(1)-b u^{\prime}(1)=\int_{0}^{1} \varrho_{1}(s) \sigma_{1}(s) d s .
\end{array}\right.
$$

Lemma 1 Assume that $h, \sigma_{0}$, and $\sigma_{1}$ are continuous functionals. If condition $\left(H_{0}\right)$ is satisfied, then problem (4) has a unique solution given by

$$
u(t)=\int_{0}^{1} G(t, s) h(s) d s+\frac{a+t}{1+a-b} \int_{0}^{1} \varrho_{1}(s) \sigma_{1}(s) d s-\frac{b-1+t}{1+a-b} \int_{0}^{1} \varrho_{0}(s) \sigma_{0}(s) d s,
$$

where $G(t, s)$ is given by

$$
G(t, s)= \begin{cases}\frac{(s+a)(1-b-t)}{1+a-b}, & 0 \leq s<t<1, \\ \frac{(1-s-b)(a+t)}{1+a-b}, & 0 \leq t<s \leq 1 .\end{cases}
$$

Here $G(t, s)$ is Green's function of $(4)$, which has the following different properties:

$$
\begin{aligned}
& \int_{0}^{1} G(t, s) d s=\frac{-(1+a-b) t^{2}+(1-2 b) t+a(1-2 b)}{2(1+a-b)}, \quad 0 \leq t \leq 1, \\
& \int_{0}^{\frac{1}{\omega}} G\left(\frac{1}{2}, s\right) d s=\frac{(1-2 b)(1+2 a \omega)}{4 \omega^{2}(1+a-b)}, \quad \omega>2, \\
& \int_{\frac{1}{\omega}}^{\frac{1}{2}} G\left(\frac{1}{2}, s\right) d s=\frac{(1-2 b)(\omega-2)[(4 a+1) \omega+2]}{16 \omega^{2}(1+a-b)}, \quad \omega>2, \\
& \int_{t_{0}}^{t_{1}} G\left(t_{1}, s\right) d s+\int_{1-t_{1}}^{1-t_{0}} G(t, s) d s=\frac{\left(t_{1}-t_{0}\right)(1-2 b)(a+t)}{2(1+a-b)}, \quad 0<t_{0}<t_{1}<\frac{1}{2}, \\
& \min _{\omega \in[0,1]} \frac{G\left(t_{0}, \omega\right)}{G\left(t_{1}, \omega\right)}=1, \quad 0<t_{0}<t_{1}<\frac{1}{2}, \quad \max _{\omega \in[0,1]} \frac{G\left(\frac{1}{2}, \omega\right)}{G(t, \omega)}=1, \quad 0<t \leq \frac{1}{2} .
\end{aligned}
$$

Define the linear operator $B: C(I) \rightarrow C(I)$ by

$$
(B u)(t)=\int_{0}^{1} \varphi(t, s) u(s) d s
$$


Lemma 2 If $\left(H_{0}\right)$ and $\left(H_{1}\right)$ are satisfied, then the operator $B$ has the following properties:

(i) $B$ is a bounded linear operator, $B(P) \subset P$;

(ii) $(I-B)$ is invertible;

(iii) $\left\|(I-B)^{-1}\right\| \leq \frac{1}{1-M}$.

Proof

(i) For all $k_{1}, k_{2} \in \mathbb{R}$ and $u_{1}(t), u_{2}(t) \in C(I)$,

$$
\begin{aligned}
B & \left(k_{1} u_{1}(t)+k_{2} u_{2}(t)\right) \\
& =\int_{0}^{1} \phi(t, s)\left[k_{1} u_{1}(t)+k_{2} u_{2}(t)\right] d s \\
& =k_{1}\left(B u_{1}\right)(t)+k_{2}\left(B u_{2}\right)(t) .
\end{aligned}
$$

By using $\left(H_{1}\right)$ and $\varphi(t, s) \leq M$ we have that

$$
|(B u)(t)| \leq M\|u\| .
$$

For $u \in P$, we have $u(s) \geq 0, s \in[0,1]$. Since $\varphi(t, s) \geq m>0$, we can obtain the following inequalities:

$$
\begin{aligned}
& (B u)(t) \geq 0 ; \quad(B u)(t)=(B u)(1-t) \quad\left(0<t<\frac{1}{2}\right) ; \\
& (B u)^{\prime \prime}(t)=-u(t)<0 ; \quad(B u)(\widetilde{t}) \leq 2 \widetilde{t} B u\left(\frac{1}{2}\right) .
\end{aligned}
$$

Then, $B(P) \subset P$.

(ii) To prove that $(I-B)$ is invertible, we just need to show that 1 is not an eigenvalue of $B$.

Since $M<1,\|B u\| \leq M\|u\|$, and thus $\sup _{u \neq 0} \frac{\|B u\|}{\|B\|} \leq M<1$.

Besides, if we suppose that 1 is an eigenvalue of $B$, then there is $u \in C(I)$ such that $B u=u$. Moreover, we can obtain that $\frac{\|B u\|}{\|B\|}=1$. So $\|B\| \geq 1$. Thus, this assumption is false, so that 1 is not an eigenvalue of $B$ and, equivalently, $(I-B)$ is invertible.

(iii) To obtain the expression for $(I-B)^{-1}$, we make use of the theory of Fredholm integral equations.

For each $t \in I, u(t)=(I-B)^{-1} x(t) \Leftrightarrow u(t)=x(t)+(B u)(t)$. We obtain

$$
u(t)=x(t)+\int_{0}^{1} \varphi(t, s) u(s) d s
$$

By using successive substitutions in (8), the condition $M<1$ implies that 1 is not an eigenvalue of the kernel $\varphi(t, s)$. So (8) has a unique continuous solution $u(t)$ for every continuous function $x(t)$.

By successive substitutions in (8) we get

$$
u(t)=x(t)+\int_{0}^{1} K(t, s) x(s) d s
$$


where the kernel $K(t, s)$ is given by

$$
K(t, s)=\sum_{j=1}^{\infty} \varphi_{j}(t, s)
$$

Here $\varphi_{j}(t, s)=\int_{0}^{1} \varphi(t, \tau) \varphi_{j-1}(\tau, s) d s, j=2,3, \ldots$, and $\varphi_{1}(t, s)=\varphi(t, s)$.

Because $|\varphi(t, s)| \leq M<1$, the series on the right in (10) is convergent. It can be easily verified that $K(t, s) \leq \frac{M}{1-M}$. So we can get

$$
(I-B)^{-1} x(t)=x(t)+\int_{0}^{1} K(t, s) x(s) d s
$$

Therefore,

$$
\begin{aligned}
\left|(I-B)^{-1} x(t)\right| & \leq|x(t)|+\frac{M}{1-M} \int_{0}^{1} x(s) d s \\
& \leq\|x\|\left(1+\frac{M}{1-M}\right) \\
& =\frac{1}{1-M}\|x\|,
\end{aligned}
$$

so that

$$
\frac{\left\|(I-B)^{-1} x\right\|}{\|x\|} \leq \frac{1}{1-M}
$$

Thus,

$$
\left\|(I-B)^{-1}\right\| \leq \frac{1}{1-M}
$$

The proof of the lemma is over.

Remark 1 Since $\varphi(t, s) \geq m$ for each $(t, s) \in I^{2}$, we analogously have $K(t, s) \geq \frac{m}{1-m}$.

Lemma 3 A function $u(t) \in P$ is a solution of (1) if and only if

$$
u(t)=\int_{0}^{1} G(t, s) f(u(s)) d s+\int_{0}^{1} \varphi(t, s) u(s) d s, \quad \text { for } t \in[0,1]
$$

Define the nonlinear operator $T: C(I) \rightarrow C(I)$ by

$$
(T u)(t)=\int_{0}^{1} G(t, s) f(u(s)) d s
$$

From (12) we obtain another form of $u(t)$,

$$
u(t)=(T u)(t)+(B u)(t) .
$$

By Lemma 2, (14) is equivalent to

$$
u(t)=(I-B)^{-1}(T u)(t) .
$$


Hence, we construct a composite operator $\Phi$ :

$$
\Phi:=(I-B)^{-1} T
$$

Lemma 4 If $u(t)$ of (12) is a cone in $P$ and $\Phi$ is as in (15), then $\Phi: P \rightarrow P$.

Proof By the above lemmas we easily get

$$
(\Phi u)(t)=\int_{0}^{1} G(t, s) f(u(s)) d s+\int_{0}^{1} K(t, s) \int_{0}^{1} G(s, \tau) f(u(\tau)) d \tau d s
$$

First, we will verify the conditions of cone $P$,

(i) if $u \in p$, then, by some properties of $G(t, s), \Phi u(t) \geq 0$,

(ii) $(\Phi u)^{\prime \prime}(t)=-f(u(s)) \leq 0,0<t<1$, that is, $\Phi u(t)$ is concave.

(iii) $\Phi u(\widetilde{t}) \geq 2 \tilde{t} \Phi u\left(\frac{1}{2}\right), \Phi u(t)=\Phi u(1-t), 0<t<\frac{1}{2}$, that is, $\Phi u(t)$ is symmetric.

This means that $\Phi u(t) \in P$, and so $\Phi: P \rightarrow P$.

\section{Main results}

To show our main result, we first assume that the following condition is established:

$$
\begin{gathered}
\left(H_{2}\right)\left(\text { i) } f(\epsilon) \leq \frac{8\left[p a \omega^{2}-l(1+2 a \omega)\right](1+a-b)(1-M)}{a(1-2 b)(\omega-2)[(4 a+1) \omega+2]}, \quad \frac{2 p}{l}<\epsilon<p ;\right. \\
\text { (ii) } f(\epsilon)>\frac{2 q(1-m)(1+a-b)}{(a+1)\left(t_{1}-t_{0}\right)(1-2 b)}, \quad q<\epsilon<\frac{q t_{1}}{t_{0}} ; \\
\text { (iii) } f(\epsilon) \leq \frac{8 l(1-M)(1+a-b)^{2}}{[2(a+b)+1]^{2}-4 a b(2 a-2 b+5)}, \quad 0<\epsilon<\frac{l}{\widetilde{2 t}}
\end{gathered}
$$

Theorem 2 Suppose that $f$ satisfies $\left(H_{2}\right)$. Let real constants $p>0, q>0$, and $l>0$ be such that $0<p<q \leq \frac{l t_{0}}{t_{1}}$. Then system (1) has three symmetric positive solutions $u_{1}(t), u_{2}(t)$, and $u_{3}(t)$ satisfying

$$
\begin{aligned}
& \max _{t \in[0, \widetilde{t}] \cup[1-\widetilde{t}, 1]} u_{i}(t) \leq l, \quad i=1,2,3, \\
& \min _{t \in\left[t_{0}, t_{1}\right] \cup\left[1-t_{1}, 1-t_{0}\right]} u_{1}(t)>q, \quad \max _{t \in\left[\frac{1}{\omega}, 1-\frac{1}{\omega}\right]} u_{2}(t)<p, \\
& \min _{t \in\left[t_{0}, t_{1}\right] \cup\left[1-t_{1}, 1-t_{0}\right]} u_{3}(t)<q, \quad \text { with } \max _{t \in\left[\frac{1}{\omega}, 1-\frac{1}{\omega}\right]} u_{3}(t)>p .
\end{aligned}
$$

Proof The following five steps are used for verifying the conditions of Theorem 1 .

(i) For all $u \in P$, from (2) and (3) we obtain $\beta(u) \leq \alpha(u),\|u\| \leq \frac{1}{2 t} \zeta(u)$.

If $u \in \overline{\mathbb{P}(\zeta, l)}$, then $\|u\| \leq \frac{1}{2 t} \zeta(u)<\frac{l}{2 t}$, and from assumption (iii) of $\left(H_{2}\right)$ we get

$$
\begin{aligned}
\zeta(\Phi u) & =\max _{t \in[0, \widetilde{t}] \cup[1-\widetilde{t}, 1]}\left\{\int_{0}^{1} G(t, s) f(u(s)) d s+\int_{0}^{1} K(t, s) \int_{0}^{1} G(s, \tau) f(u(\tau)) d \tau d s\right\} \\
& \leq\left(1+\frac{M}{1-M}\right) \int_{0}^{1} G(\widetilde{t}, s) f(u(s)) d s \\
& \leq \frac{1}{1-M} \frac{8 l(1-M)(1+a-b)^{2}}{[2(a+b)+1]^{2}-4 a b(2 a-2 b+5)} \int_{0}^{1} G(\widetilde{t}, s) d s
\end{aligned}
$$




$$
\begin{aligned}
& \left.\leq \frac{1}{1-M} \frac{2 l(1-M)(1+a-b)}{-(1+a-b) \widetilde{t}^{2}+(1-2 b) \widetilde{t}+a(1-2 b)} \int_{0}^{1} G \widetilde{t}, s\right) d s \\
& =l
\end{aligned}
$$

Thus, $\Phi: \overline{\mathbb{P}(\zeta, l)} \rightarrow \overline{\mathbb{P}(\zeta, l)}$, and we immediately get that

$$
\begin{aligned}
& \left\{u \in \mathbb{P}\left(\zeta, \rho, \beta, q, \frac{q t_{1}}{t_{0}}, l\right): \beta(u)>q\right\} \neq \emptyset \text { and } \\
& \left\{u \in \mathbb{Q}\left(\zeta, \alpha, \chi, \frac{2 p}{l}, p, l\right): \alpha(u)<p\right\} \neq \emptyset .
\end{aligned}
$$

(ii) If $u \in \mathbb{Q}(\zeta, \alpha, p, l)$ with $\chi(\Phi u)<\frac{2 p}{l}$, then we obtain

$$
\begin{aligned}
\alpha(\Phi u) & =\max _{t \in\left[\frac{1}{\omega}, 1-\frac{1}{\omega}\right]}\left\{\int_{0}^{1} G(t, s) f(u(s)) d s+\int_{0}^{1} K(t, s) \int_{0}^{1} G(s, \tau) f(u(\tau)) d \tau d s\right\} \\
& \leq\left(1+\frac{M}{1-M}\right) \int_{0}^{1} G\left(\frac{1}{2}, s\right) f(u(s)) d s \\
& =\frac{1}{1-M} \int_{0}^{1} \frac{G\left(\frac{1}{2}, s\right)}{G\left(\frac{1}{\omega}, s\right)} G\left(\frac{1}{\omega}, s\right) f(u(s)) d s \\
& \leq \frac{1}{1-M} \int_{0}^{1} G\left(\frac{1}{\omega}, s\right) f(u(s)) d s \\
& \leq \frac{1-m}{1-M} \chi(\Phi u) \\
& <\frac{1-m}{1-M} \frac{2 p}{l} \\
& <p .
\end{aligned}
$$

(iii) If $u \in \mathbb{Q}\left(\zeta, \alpha, \chi, \frac{2 p}{l}, p, l\right)$, then from assumption (i) and (iii) of $\left(H_{2}\right)$ we get

$$
\begin{aligned}
\alpha(\Phi u)= & \max _{t \in\left[\frac{1}{\omega}, 1-\frac{1}{\omega}\right]}\left\{\int_{0}^{1} G(t, s) f(u(s)) d s+\int_{0}^{1} K(t, s) \int_{0}^{1} G(s, \tau) f(u(\tau)) d \tau d s\right\} \\
\leq & \left(1+\frac{M}{1-M}\right) \int_{0}^{1} G\left(\frac{1}{2}, s\right) f(u(s)) d s \\
= & \frac{1}{1-M} \int_{0}^{1} G\left(\frac{1}{2}, s\right) f(u(s)) d s \\
= & \frac{2}{1-M}\left\{\int_{0}^{\frac{1}{\omega}} G\left(\frac{1}{2}, s\right) f(u(s)) d s+\int_{\frac{1}{\omega}}^{\frac{1}{2}} G\left(\frac{1}{2}, s\right) f(u(s)) d s\right\} \\
\leq & \frac{2}{1-M}\left\{\frac{8 l(1-M)(1+a-b)^{2}}{[2(a+b)+1]^{2}-4 a b(2 a-2 b+5)} \int_{0}^{\frac{1}{\omega}} G\left(\frac{1}{2}, s\right) d s\right. \\
& \left.+\frac{8\left[p a \omega^{2}-l(1+2 a \omega)\right](1+a-b)(1-M)}{a(1-2 b)(\omega-2)[(4 a+1) \omega+2]} \int_{\frac{1}{\omega}}^{\frac{1}{2}} G\left(\frac{1}{2}, s\right) d s\right\} \\
\leq & \frac{2}{1-M}\left\{\frac{2 l(1-M)(1+a-b)}{a(1-2 b)} \int_{0}^{\frac{1}{\omega}} G\left(\frac{1}{2}, s\right) d s\right.
\end{aligned}
$$




$$
\begin{aligned}
& \left.+\frac{8\left[p a \omega^{2}-l(1+2 a \omega)\right](1+a-b)(1-M)}{a(1-2 b)(\omega-2)[(4 a+1) \omega+2]} \times \int_{\frac{1}{\omega}}^{\frac{1}{2}} G\left(\frac{1}{2}, s\right) d s\right\} \\
= & p .
\end{aligned}
$$

(iv) If $u \in \mathbb{Q}(\zeta, \beta, q, l)$ with $\rho(\Phi u)>\frac{q t_{1}}{t_{0}}$, then we get

$$
\begin{aligned}
\beta(\Phi u)= & \min _{t \in\left[t_{0}, t_{1}\right] \cup\left[1-t_{1}, 1-t_{0}\right]}\left\{\int_{0}^{1} G(t, s) f(u(s)) d s\right. \\
& \left.+\int_{0}^{1} K(t, s) \int_{0}^{1} G(s, \tau) f(u(\tau)) d \tau d s\right\} \\
\geq & \left(1+\frac{m}{1-m}\right) \int_{0}^{1} G\left(t_{0}, s\right) f(u(s)) d s \\
= & \frac{1}{1-m} \int_{0}^{1} \frac{G\left(t_{0}, s\right)}{G\left(t_{1}, s\right)} G\left(t_{1}, s\right) f(u(s)) d s \\
\geq & \frac{1}{1-m} \int_{0}^{1} G\left(t_{1}, s\right) f(u(s)) d s \\
\geq & \frac{1-M}{1-m} \rho(\Phi u) \\
> & \frac{1-M}{1-m} \frac{q t_{1}}{t_{0}} \\
> & q .
\end{aligned}
$$

(v) If $u \in \mathbb{Q}\left(\zeta, \rho, \beta, q, \frac{q t_{1}}{t_{0}}, l\right)$, then from assumption (ii) of $\left(H_{2}\right)$ we get

$$
\begin{aligned}
\beta(\Phi u)= & \min _{t \in\left[t_{0}, t_{1}\right] \cup\left[1-t_{1}, 1-t_{0}\right]}\left\{\int_{0}^{1} G(t, s) f(u(s)) d s\right. \\
& \left.+\int_{0}^{1} K(t, s) \int_{0}^{1} G(s, \tau) f(u(\tau)) d \tau d s\right\} \\
\geq & \left(1+\frac{m}{1-m}\right) \int_{0}^{1} G\left(t_{0}, s\right) f(u(s)) d s \\
= & \frac{1}{1-m} \int_{0}^{1} G\left(t_{0}, s\right) f(u(s)) d s \\
> & \frac{1}{1-m}\left\{\int_{t_{0}}^{t_{1}} G\left(t_{1}, s\right) f(u(s)) d s+\int_{1-t_{1}}^{1-t_{0}} G\left(t_{0}, s\right) f(u(s)) d s\right\} \\
\geq & \frac{1}{1-m} \cdot \frac{2 q(1-m)(1+a-b)}{\left(a+t_{0}\right)\left(t_{1}-t_{0}\right)(1-3 b)}\left\{\int_{t_{0}}^{t_{1}} G\left(t_{0}, s\right) d s+\int_{1-t_{1}}^{1-t_{0}} G\left(t_{0}, s\right) d s\right\} \\
= & q .
\end{aligned}
$$

\section{Example}

In the section, we provide a simple example illustrating the application of our main results. 
Example 1 Let $a=\frac{3}{2}, b=-\frac{1}{2}, \varrho_{0}=\varrho_{1}=\frac{1}{3}$, and $f(u(t))=u(t)$, Then $(1)$ turns to the following equation:

$$
\left\{\begin{array}{l}
-u^{\prime \prime}(t)=u(t), \quad t \in I \\
u(0)-\frac{3}{2} u^{\prime}(0)=\frac{1}{3} \int_{0}^{1} u(s) d s \\
u(1)+\frac{1}{2} u^{\prime}(1)=\frac{1}{3} \int_{0}^{1} u(s) d s
\end{array}\right.
$$

Because of the main result in Section 4, we have the following result.

Corollary 1 Choose $p=\frac{1}{3}, q=2, l=3, t_{0}<t_{1}$, and $\omega>2$ such that $0<p<q \leq \frac{l t_{0}}{t_{1}}$. Then the boundary value problem (16) has three symmetric positive solutions $u_{1}(t), u_{2}(t)$ and $u_{3}(t)$ satisfying

$$
\begin{aligned}
& \max _{t \in[0, \widetilde{t}] \cup[1-\tilde{t}, 1]} u_{i}(t) \leq 3, \quad i=1,2,3, \\
& \min _{t \in\left[t_{0}, t_{1}\right] \cup\left[1-t_{1}, 1-t_{0}\right]} u_{1}(t)>2, \quad \max _{t \in\left[\frac{1}{\omega}, 1-\frac{1}{\omega}\right]} u_{2}(t)<\frac{1}{3}, \\
& \min _{t \in\left[t_{0}, t_{1}\right] \cup\left[1-t_{1}, 1-t_{0}\right]} u_{3}(t)<2, \quad \text { with } \max _{t \in\left[\frac{1}{\omega}, 1-\frac{1}{\omega}\right]} u_{3}(t)>\frac{1}{3} .
\end{aligned}
$$

Proof Now, we only verify conditions of $\left(H_{2}\right)$ in Theorem 2.

(i) For $\frac{2}{9}<u(t)<\frac{1}{3}$,

$$
f(u(t)) \leq \frac{8\left(\omega^{2}-3 \omega-6\right)}{21\left(\omega^{2}-\frac{12}{7} \omega-\frac{4}{7}\right)}, \quad \text { and, obviously, } \quad \frac{1}{3} \leq \frac{8\left(\omega^{2}-3 \omega-6\right)}{21\left(\omega^{2}-\frac{12}{7} \omega-\frac{4}{7}\right)}
$$

(ii) For $2<u(t)<\frac{2 t_{1}}{t_{0}}$,

$$
f(u(t))>\frac{8}{5\left(t_{1}-t_{0}\right)}, \quad \text { and } \quad 2>\frac{8}{5\left(t_{1}-t_{0}\right)}
$$

(iii) For $0<u(t)<\frac{3}{2 t}$,

$$
f(u(t)) \leq 4, \quad \text { and } \quad \frac{3}{2 t} \leq 4
$$

We can complete the proof according Theorem 2.

\section{Competing interests}

The authors have no any competing interests.

\section{Authors' contributions}

Zhiying Tong wrote the first draft of a paper, revising and editing. Wei Ding is in charge of the topic, the method, revising and editing.

\section{Acknowledgements}

The authors are very grateful to the reviewers and the editor for their valuable suggestions and comments. This work was supported by National Natural Science Foundation of China (No. 11431008, 11271261) and Shanghai Municipal Education Commission (No. 14ZZ12). 


\section{References}

1. Boucherif, A, Henderson, J: Positive solutions of second order boundary value problems with sign changing Carathéodory nonlinearities. Electron. J. Qual. Theory Differ. Equ. 2006, 7 (2006)

2. Cac, NP, Fink, AM, Gatica, JA: Nonnegative solutions of quasilinear elliptic boundary value problems with nonnegative coefficients. J. Math. Anal. Appl. 206, 1-9 (1997)

3. de Figueiredo, DG, Lions, PL, Nussbaum, RD: A priori estimates and existence of positive solutions of semilinear elliptic equations. J. Math. Pures Appl. 61, 41-63 (1982)

4. Gallardo, JM: Second order differential operators with integral boundary conditions and generation of semigroups. Rocky Mt. J. Math. 30, 1265-1292 (2000)

5. Guo, D, Lakshmikantham, V: Nonlinear Problems in Abstract Cones. Academic Press, San Diego (1988)

6. Gupta, CP: A note on a second order three-point boundary value problem. J. Math. Anal. Appl. 186, $277-281$ (1994)

7. Ionkin, NI: Solution of a boundary value problem in heat conduction theory with nonlocal boundary conditions. Differ. Equ. 13, 294-304 (1977)

8. Karakostas, GL, Tsamatos, PC: Multiple positive solutions of some Fredholm integral equations arisen from nonlocal boundary-value problems. Electron. J. Differ. Equ. 2002, 30 (2002)

9. Keller, JB: Some positone problems suggested by nonlinear hear generation. In: Bifurcation Theory and Nonlinear Eigenvalue Problems, pp. 217-255. Benjamin, Elmsford (1969)

10. Liang, $\mathrm{SH}$, Zhang, JH: Existence of three positive solutions of three-order with $m$-point impulsive boundary value problems. Acta Appl. Math. 110, 1265-1292 (2010)

11. Lomtatidze, A, Malaguti, L: On a nonlocal boundary-value problems for second order nonlinear singular differential equations. Georgian Math. J. 7, 133-154 (2000)

12. Zhang, XM, Feng, MQ, Ge, WG: Existence of solutions of boundary value problems with integral boundary conditions for second-order impulsive integro-differential equations in Banach spaces. J. Comput. Appl. Math. 233, 1915-1926 (2010)

13. Zhao, JF, Wang, PG, Ge, WG: Existence and nonexistence of positive solutions for a class of third order BVP with integral boundary conditions in Banach spaces. Commun. Nonlinear Sci. Numer. Simul. 16, 402-413 (2011)

14. Boucherif, A: Second-order boundary value problems with integral boundary conditions. Nonlinear Anal. 70, 364-371 (2009)

15. Hayasida, K, Nakatani, M: On radially symmetric positive solutions of a semilinear elliptic equation in hyperbolic space. Math. Jpn. 40(3), 561-584 (1994)

16. Avery, RI, Davis, JM, Henderson, J: Three symmetric positive solutions for Lidstone problems by a generalization of the Leggett-Williams theorem. Electron. J. Differ. Equ. 2000, 40 (2000) 15 pp.

17. Agarwal, RP, O'Regan, D: A generalization of the Petryshyn-Leggett-Williams fixed point theorem with applications to integral inclusions. Appl. Math. Comput. 123(2), 263-274 (2001)

18. Agarwal, RP, Ravi, $\mathrm{P}, \mathrm{O}^{\prime}$ Regan, D: A fixed point theorem of Leggett-Williams type with applications to single- and multivalued equations. Georgian Math. J. 8(1), 13-25 (2001)

19. Agarwal, RP, O'Regan, D: Existence of three solutions to integral and discrete equations via the Leggett Williams fixed point theorem. Rocky Mt. J. Math. 31(1), 23-35 (2001)

20. Abdulkadir, D: On the existence of positive solutions for the second-order boundary value problem. Appl. Math. Lett. 49, 107-112 (2015)

21. Avery, Rl: A generalization of the Leggett-Williams fixed point theorem. Math. Sci. Res. Hot-Line 3, 9-14 (1999)

\section{Submit your manuscript to a SpringerOpen ${ }^{\circ}$ journal and benefit from:}

- Convenient online submission

Rigorous peer review

- Immediate publication on acceptance

Open access: articles freely available online

- High visibility within the field

- Retaining the copyright to your article 